\title{
Radiation-Induced Segregation in Multicomponent Alloys: Effect of Particle Type
}

\author{
G. S. Was* and T. Allen ${ }^{\dagger}$ \\ ${ }^{*}$ Department of Nuclear Engineering, *Department of Materials Science and Engineering, \\ University of Michigan, Ann Arbor, MI 48109
}

The problem of irradiation-assisted stress-corrosion cracking (IASCC) in reactor cores is currently being addressed using different types of particle irradiation (electrons, protons, and heavy ions) to study the effect of neutron damage. The effect of radiation damage of greatest interest to the IASCC problem is the segregation of impurities or redistribution of alloying elements in the vicinity of the grain boundary. Differences among the types of irradiation include particle type, temperature, dose, and dose rate. The different particle type results in different "effective" displacement rates due to fundamental differences in the radiation damage state. The effect of displacement efficiency is incorporated into existing models for radiation-induced segregation and compared with experimentally determined values. Comparisons between theory and experiment were made using neutron-, proton-, and ion-irradiated stainless steels. Results showed that the measured chromium depletion profile is generally narrower and shallower than that calculated from theory. Agreement between experiment and model was better in almost all cases when the particle efficiency was taken into account. Agreement was best for proton-irradiated steels, and less so for neutron and heavy ion-irradiated steels. The origins of this difference are probably due to spatial and depth resolution of the scanning transmission electron microscopy and Auger electron spectrometry measurement techniques, respectively, the lack of knowledge of the material parameters in modeling, and the uncertainty in the true displacement rate. Nevertheless, beyond $2 \mathrm{~nm}$ from the grain boundary, the shape of the experimental and calculated profiles agree reasonably well.

\section{INTRODUCTION}

Light water reactors are becoming increasingly plagued by the failure of core internals from irradiation-assisted stress-corrosion cracking (IASCC) [1]. IASCC refers to the acceleration of the stress-corrosion cracking process by irradiation. Broadly speaking, IASCC can result from irradiation by gammas, neutrons, electrons or ions, and any material-environment combination in which SCC is known to occur. However, in a practical sense, the problem addresses the accel- erated intergranular cracking process in austenitic alloys in light water reactor cores by neutron irradiation. Intergranular stresscorrosion cracking (IGSCC) in austenitic alloys in reactors is a well-established and much studied phenomenon. IGSCC of these alloys can occur without the aid of irradiation, and isolating the component of the observed cracking due to irradiation elevates the difficulty of the problem. The neutron effect is characterized by an increase in intergranular cracking and a decrease in ductility at a neutron fluence above $\sim 5 \times 10^{20} \mathrm{n} / \mathrm{cm}^{2}$ 
(E $>1 \mathrm{MeV}$ ), which corresponds to about 1 displacement per atom (dpa). Changes to the alloy could involve microstructural changes, microchemical changes, compositional changes by transmutation, and interaction among these changes. This must be interfaced with changes in water chemistry and corrosion potential. Accounting for the role of stress along with changes to the environment and alloy, the problem quickly becomes multidimensional.

IASCC has been occurring for over 20 years in core components made of austenitic iron- and nickel-based alloys in both pressurized water reactors (PWRs) and boiling water reactors (BWRs) [2]. Early failures occurred in 304, 304L, and 347 SS fuel rod cladding in BWRs [3-5]. More recently, IASCC has been suspected in failures of 304 SS fuel cladding in the Connecticut Yankee Reactor (PWR) [6]. The problem has also been found in 304 SS control rod absorber tubes, fuel bundle cap screws, control rod blade handles, sheaths and follower rivets, plate-type control blades, and instrument dry tubes in BWRs [2]. It is becoming increasingly evident that the problem is widespread without regard to environment or material composition and that tens or hundreds of core components may be susceptible to this form of degradation. A recent review by Andresen et al. [2] identified some 17 components, spanning 6 iron-base or nickel-base alloys and 4 reactor designs. Given that a threshold fluence has been identified, many of the susceptible components may just now be coming to our attention, and the extent of the problem may continue to expand. This also implies that the observed behavior is at least in part due to irradiation-induced changes in the alloy.

With regard to the mechanism of IASCC, most of the recent attention has been focused on the issue of radiation-induced segregation (RIS) of impurities to grain boundaries as being the main cause of the "weakening" of the grain boundaries. This segregation process is fundamentally different from thermal segregation in that it is driven by the flux of radiation-produced defects to sinks such as the free surface, grain boundaries, and other interfaces [7]. Vacancies and interstitials are the basic defects produced by irradiation and can reach concentrations that are orders of magnitude greater than the thermal equilibrium concentration. Diffusion of solutes by vacancy or interstitial mechanisms is then accelerated by the elevated concentration of these defects. As a function of temperature, segregation peaks at intermediate temperatures because a lack of mobility shuts down the process at low temperatures, and recombination dominates at high temperatures where defect concentrations approach their thermal equilibrium values. If the relative participation of alloying elements in the defect fluxes is not the same as the relative concentration of the alloying elements, then a net transport of the constituents to or from the sinks will occur (Fig. 1). The result will be either a buildup or a depletion of alloying elements at the sinks-principally grain boundaries and free surfaces. The magnitude of the buildup/depletion is dependent upon several factors, such as whether a constituent migrates more rapidly by one defect mechanism or another, and the binding between solutes and defects.

A common characteristic of RIS profiles is their narrowness, often confined to within $5-10 \mathrm{~nm}$ of the grain boundary. Irradiation of stainless steels results in the redistribution of the major alloying elements and the segregation of impurities. RIS studies have shown that Ni segregates to the grain boundaries while $\mathrm{Cr}$ and $\mathrm{Fe}$ are depleted at the boundaries. With regard to impurities, P, S, and $\mathrm{Si}$ are known to become enriched, and Mo and Ti are depleted at the grain boundaries. Interestingly, the directions of segregation are consistent with an atomic volume effect in which the subsized solute migrates preferentially with the interstitial flux, and the oversized solute participates preferentially in the vacancy flux.

Thus far, investigations of IASCC are being conducted on irradiated core components removed from operating power reactors or samples inserted into test reactors. Experiments consist of determining the grain boundary composition profile and the sus- 

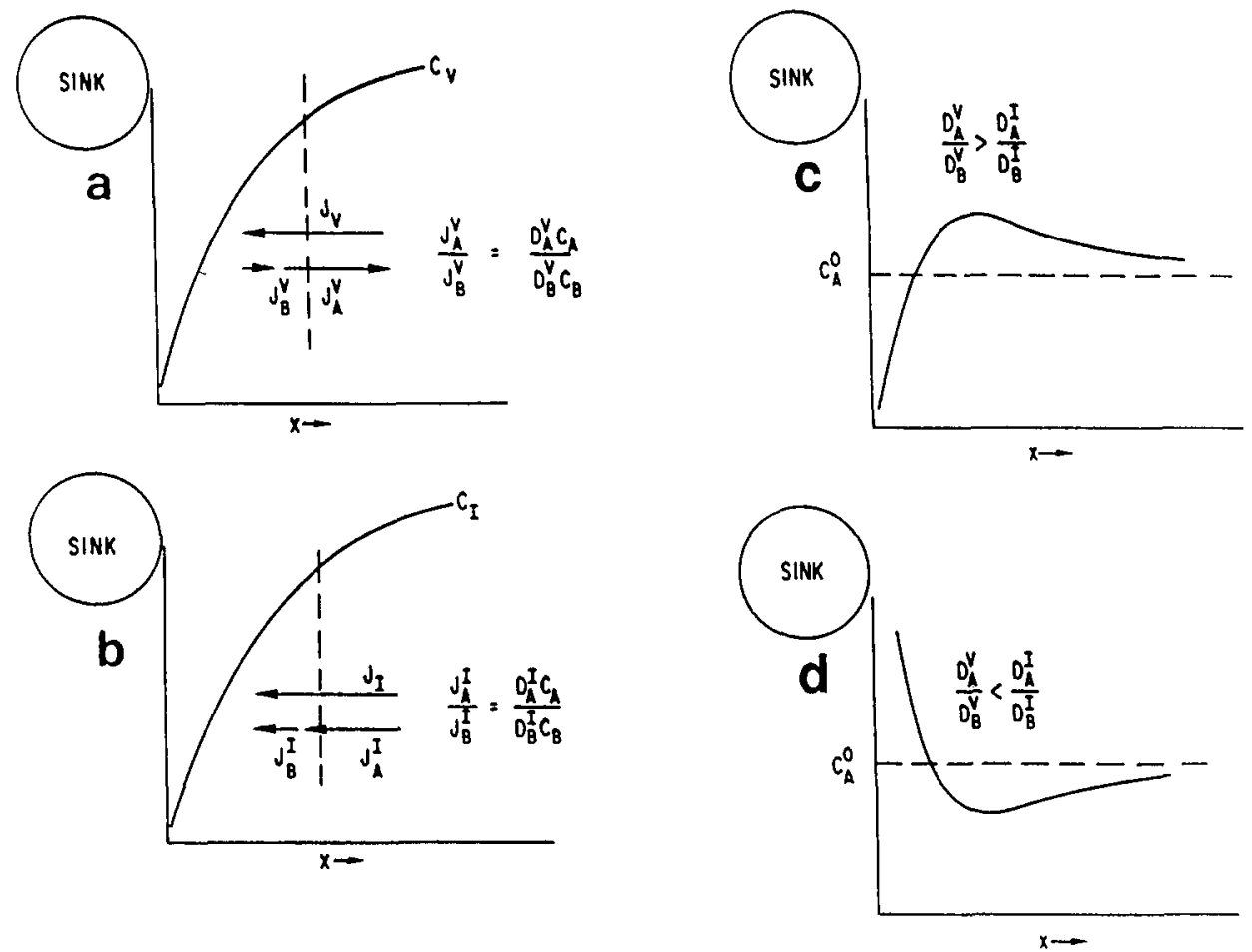

FIG. 1. Schematic of inverse Kirkendall effects induced by (a) vacancy flux and (b) interstitial flux, and (c and d) the effect of diffusion coefficients on the depth distribution of A atoms in an AB alloy (from Okamoto and Rehn [7]).

ceptibility to SCC in postirradiation analyses and tests, respectively, in a reactorlike environment. However, the temperature and radiation damage history of components in power reactors is inherently uncontrolled. In addition, due to the high level of residual radioactivity, these experiments can consume years and require substantial financial resources. Because of these factors, investigators have turned to other forms of irradiation that can be done in a well-controlled environment, require less irradiation time, and result in minimal residual radioactivity. These include heavy ions, protons, and electrons. The first question to resolve is the measure of radiation effect. In the IASCC problem, concern has centered about the effect of radiation on inducing the segregation of deleterious species to grain boundaries that then cause embrittlement or enhance the IGSCC process. The appropriate measure of the radiation effect in this case would then be the impurity concentration at the grain boundary, or the change in major element composition at the grain boundary. This is a measurable quantity, either by Auger electron spectrometry (AES) or energy-dispersive spectroscopy via scanning transmission electron microscopy (STEM-EDS). Hence, a specific and measurable effect of radiation can be determined for both neutron and ion irradiation experiments.

A problem arises in the comparison of results from neutron and charged particle irradiation experiments in that the form of the damage state for each particle type is fundamentally different. There is also a problem in determining how an ion irradiation translates into the environment describing neutron irradiation. That is, what are the irradiation conditions required for ion irradiation to yield the same measure of radiation effect as that for neutron irradiation? This is the key question in studying IASCC, for in a postirradiation test program, it is only the final state of the material that is important in the determination of equivalence and not the path taken. As such, one could probe 
(with a given ion) the temperature, dose, and dose rate space until a coordinate is identified that produces the same measure of radiation effect as neutron irradiation. However, we are interested in a more orderly approach to the problem. In order to determine a priori the conditions of the ion experiment needed to produce the same measure of radiation effect as neutron irradiation, we must be able to account for the differences between the two experiments. These differences are fourfold: particle type, temperature, dose, and dose rate. The first of these differences presents its own unique set of problems, while the remaining differences can be resolved in the temporal description of the radiation damage process. It is the objective of this paper to compare the development of the grain boundary microchemistry for different particle irradiations. Specifically, this paper will focus on the issue of accounting for differences in particle type as they affect the radiation-induced segregation profile of chromium at the grain boundary of the irradiated material. (Grain boundary chromium has long been identified as a cause of IGSCC in unirradiated, thermally treated stainless steels in reactor cores, and is a prime candidate for the explanation of IASCC.) This will be done by modeling the RIS process for the different particle types and irradiation conditions. Model results will then be compared with experiments to determine the effectiveness of the model in simulating the processes occurring during irradiation. This treatment will be preceded by short discussions of RIS, particle type and efficiency, and experiments for measuring RIS.

\section{METHODOLOGY AND EXPERIMENT}

\section{MODELING RADIATION-INDUCED SEGREGATION}

Because our interests are on the segregation behavior in ternary $\mathrm{Fe}-\mathrm{Cr}-\mathrm{Ni}$ alloys, the framework of the phenomenological model for ternary alloys will be briefly described. The following description is a condensation of that given by Lam et al. [8] for a ternary alloy whose components $A, B$, and $C$ are present in concentrations $C_{A}, C_{B}$, and $C_{C}$ (number of atoms per unit volume). Vacancy and interstitial concentrations created during irradiation vary with time according to:

$$
\begin{aligned}
& \frac{\partial C_{v}}{\partial t}=-\nabla \cdot J_{v}+K-R, \\
& \frac{\partial C_{i}}{\partial t}=-\nabla \cdot J_{i}+K-R,
\end{aligned}
$$

where $\nabla \cdot J_{v}$ and $\nabla \cdot J_{i}$ are the divergences of the vacancy and interstitial fluxes at the defect sinks, and $K$ and $R$ are the local total rates of production and mutual recombination, respectively, of point defects. The defect fluxes are partitioned into those occurring via $\mathrm{A}, \mathrm{B}$, and $\mathrm{C}$ atoms in the alloy according to:

$J_{i}=J_{i}^{A}+J_{i}^{B}+J_{i}^{C}$,
$J_{v}=J_{v}^{A}+J_{v}^{B}+J_{v}^{C}$,

where the subscripts indicate the species of flux, and the superscripts indicate the complementary species by which the flux occurs. The partial interstitial fluxes are in the same direction as the corresponding atom fluxes, while the partial vacancy fluxes are in the opposite direction to the atom fluxes,

$\begin{array}{ll}J_{i}^{A}=J_{A}^{i}, & J_{i}^{B}=J_{B}^{i}, \quad J_{i}^{C}=J_{C}^{i}, \\ J_{v}^{A}=-J_{A}^{v}, & J_{v}^{B}=-J_{B}^{v}, \quad J_{v}^{C}=-J_{C}^{v}\end{array}$

and Eq. (2a) and (2b) can be written as

$J_{i}=J_{A}^{i}+J_{B}^{i}+J_{C}^{i}$,
$J_{v}=-\left(J_{A}^{v}+J_{B}^{v}+J_{C}^{v}\right)$.

These equations express the coupling between defect and atom fluxes across any fixed lattice plane. In general, the partitioning of the interstitial and vacancy fluxes via $A, B$, and $C$ atoms is not in the same proportion as the atom fractions in the alloy. Interstitials may preferentially migrate by one atom type, while vacancies preferentially exchange with another atom type. This preferential coupling of defect and atom fluxes is the physical origin of radiation-induced segregation. 
As with the defect compositions, the alloy composition in time and space can be described by the conservation equations:

$\frac{\partial C_{A}}{\partial t}=-\nabla \cdot J_{A}$,

$\frac{\partial C_{B}}{\partial t}=-\nabla \cdot I_{B}$

$\frac{\partial C_{C}}{\partial t}=-\nabla \cdot J_{C}$,

where $J_{A}, J_{B}$, and $J_{C}$ are the total fluxes of the alloying elements, which can be partitioned into partial fluxes occurring by vacancies and interstitials,

$J_{A}=J_{A}^{i}+J_{A}^{v}$,

$J_{B}=J_{B}^{i}+J_{B}^{v}$,

$J_{C}=J_{C}^{i}+J_{C}^{v}$.

The defect and atom fluxes are expressed in terms of the concentration gradients of the different species,

$J_{k}^{i}\left(\equiv J_{i}^{k}\right)=-D_{k}^{i} \alpha \nabla C_{k}-D_{i}^{k} \nabla C_{i}$,

$J_{k}^{v}\left(\equiv-J_{v}^{k}\right)=-D_{k}^{v} \alpha \nabla C_{k}+D_{v}^{k} \nabla C_{v}$

where $k=A, B$, or $C, \alpha$ is the thermodynamic factor that relates the concentration gradient to the chemical potential gradient of atoms, and $D_{k}^{i}, D_{k}^{v}, D_{i}^{k}$, and $D_{v}^{k}$ are the partial diffusion coefficients of atoms $k$ by interstitials, and vacancies, and of interstitials and vacancies by atoms, respectively. The partial diffusion coefficients have the form:

$D_{k}^{i}=d_{k j} N_{j}$ and $D_{j}^{k}=d_{k j} N_{k}$,

where $j=i$ or $v, N_{j}=\Omega C_{j}$ and $N_{k}=\Omega C_{k}$ are the atomic fractions of defects and of $k$ atoms, respectively, $\Omega$ is the average atomic volume in the alloy, and $d_{k j}$ are the diffusion coefficients for conjugate atom-defect pairs $k j$,

$d_{k j}=\frac{1}{6} \lambda_{k}^{2} z_{k} v_{k j}^{e f f}$.

Here $\lambda_{k}$ is the jump distance, $z_{k}$ is the coordination number, and $v_{k j}^{\text {eff }}$ is the effective jump or exchange frequency of the pair. The total diffusion coefficients for interstitials and vacancies are defined as:
$D_{i}=\sum_{k} d_{k i} N_{k}$

$D_{v}=\sum_{k} d_{k v} N_{k}$

and for atoms:

$D_{k}=d_{k i} N_{i}+d_{k v} N_{v}$.

From Eqs. (4), (6), (7), (8), and (10), the defect and atom fluxes with respect to a coordinate system fixed on the crystal lattice are:

$$
\begin{aligned}
J_{i}= & -\left(d_{A i}-d_{C i}\right) \Omega C_{i} \alpha \nabla C_{A} \\
& -\left(d_{B i}-d_{C i}\right) \Omega C_{i} \alpha \nabla C_{B} \\
& -D_{i} \nabla C_{i}, \\
J_{v}= & \left(d_{A v}-d_{C v}\right) \Omega C_{v} \alpha \nabla C_{A} \\
& +\left(d_{B v}-d_{C v}\right) \Omega C_{v} \alpha \nabla C_{B} \\
& -D_{v} \nabla C_{v}, \\
J_{A}= & -D_{A} \alpha \nabla C_{A}+d_{A v} \Omega C_{A} \nabla C_{v} \\
& -d_{A i} \Omega C_{A} \nabla C_{i}, \\
J_{B}= & -D_{B} \alpha \nabla C_{B}+d_{B v} \Omega C_{B} \nabla C_{v} \\
& -d_{B i} \Omega C_{B} \nabla C_{i}, \\
J_{C}= & -D_{C} \alpha \nabla C_{C}+d_{C v} \Omega C_{C} \nabla C_{v} \\
& -d_{C i} \Omega C_{C} \nabla C_{i} .
\end{aligned}
$$

Small perturbations arising from the presence of point defects are neglected so that $C_{A}+C_{B}+C_{C}=\Omega^{-1}$ and $\nabla C_{C}=-\left(\nabla C_{A}+\right.$ $\nabla C_{B}$ ). Of the five flux equations (11a-e), only four are independent because the defect and atom fluxes across a marker plane must balance:

$J_{A}+J_{B}+J_{C}=J_{i}-J_{v}$.

A system of four coupled partial differential equations describing the space and time dependence of the atoms and defects in the solid is determined by substituting the defect and atom fluxes given by Eqs. (11) into Eqs. (1) and (5),

$$
\begin{aligned}
\frac{\partial C_{v}}{\partial t}=\nabla \cdot[ & -\left(d_{A v}-d_{C v}\right) \Omega C_{v} \boldsymbol{\alpha} \nabla C_{A} \\
& -\left(d_{B v}-d_{C v}\right) \Omega C_{v} \boldsymbol{\alpha} \nabla C_{B} \\
& \left.+D_{v} \nabla C_{v}\right]+K-R, \\
\frac{\partial C_{i}}{\partial t}=\nabla \cdot[ & \left(d_{A i}-d_{C i}\right) \Omega C_{i} \boldsymbol{\alpha} \nabla C_{A} \\
& +\left(d_{B i}-d_{C i}\right) \Omega C_{i} \boldsymbol{\alpha} \nabla C_{B} \\
& \left.+D_{i} \nabla C_{i}\right]+K-R,
\end{aligned}
$$




$$
\begin{gathered}
\frac{\partial C_{A}}{\partial t}=\nabla \cdot\left[\begin{array}{c}
D_{A} \alpha \nabla C_{A} \\
\left.+\Omega C_{A}\left(d_{A i} \nabla C_{i}-d_{A v} \nabla C_{v}\right)\right]
\end{array}\right. \\
\begin{aligned}
\frac{\partial C_{B}}{\partial t}=\nabla \cdot & {\left[D_{B} \alpha \nabla C_{B}\right.} \\
& \left.+\Omega C_{B}\left(d_{B i} \nabla C_{i}-d_{B v} \nabla C_{v}\right)\right]
\end{aligned}
\end{gathered}
$$

Numerical solutions of Eqs. (13a)-(13d) are obtained for a planar sample under irradiation with energetic particles with the aid of the GEAR package of subroutines [9]. In studying grain boundary segregation, the grain boundary was equated to a free surface and the calculations were performed for only a single grain, taking advantage of the symmetry of the problem. The initial conditions were the thermodynamic equilibrium of the alloy. Conditions at the boundary were defined as follows: At the grain center, all concentration gradients were set equal to zero. At the grain boundary, the concentrations of interstitials and vacancies were fixed at their thermal equilibrium values. The grain boundary atom concentrations were determined by the conservation of the numbers of atoms in the specimen. Atom concentrations were assumed to be initially uniform. Parameters used in the calculation of segregation in $\mathrm{Fe}-\mathrm{Cr}-\mathrm{Ni}$ alloys are given in Lam et al. [8] for the Lam model and in Perks et al. [10] for the Perks model as coded by Simonen [11].

\section{ACCOUNTING FOR PARTICLE TYPE AND EFFICIENCY}

The first problem in determining the equivalence between the measure of radiation effect in charged particle and neutron irradiation is the use of a common dose unit. The basic (measurable) dose unit for neutron irradiation is $\mathrm{n} / \mathrm{cm}^{2}$ greater than some energy threshold $(E>x \mathrm{MeV})$, where $x$ is the energy threshold. For charged particles, it is the integrated current or charge, $\mathrm{Q} / \mathrm{cm}^{2}$. The particle beam community is accustomed to reporting dose in units of dpa and dose rate as dpa/s using one of several models for the determination of dpa. Although somewhat more complicated, due to the existence of an energy spectrum rather than a mono- energetic ion beam, the same conversion can be made from $\mathrm{n} / \mathrm{cm}^{2}(E>x \mathrm{MeV})$, although it is seldom done. Radiation damage, in the form of the number of displacements per atom, is generally determined using some form of the Kinchen-Pease (K-P) displacement model [12] given by:

$v(T)=0.8 T_{\text {damage }} / 2 E_{d}$,

where $T_{\text {damage }}$ is the energy transferred from the incoming particle to the struck atom, and $E_{d}$ is the displacement energy, that is, that energy needed to displace the struck atom from its lattice position. However, the "ballistically" determined value of dpa calculated using such a displacement model is not the appropriate unit to be used for dose comparisons between particle types. The reason is the difference in the primary damage state among different particle types.

Figure 2 shows the relationship between damage morphology (as quantified by the
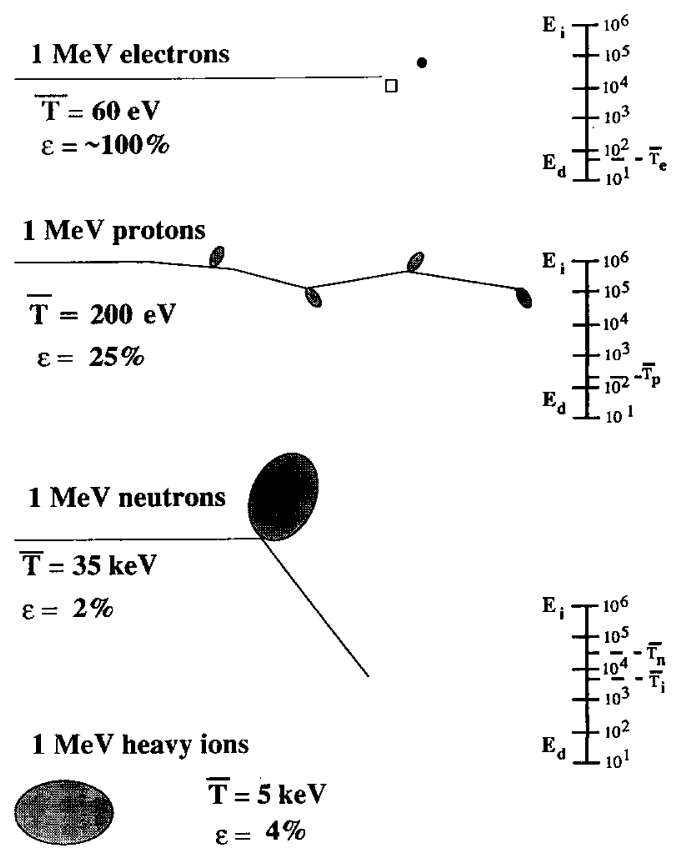

FIG. 2. Description of damage morphologies for irradiation with various particles of the same energy. $T$ is the average energy transfer per PKA and $\varepsilon$ is the efficiency of producing freely migrating defects available to affect radiation-induced segregation. 
average energy transfer in a PKA collision) and the displacement efficiency for various particle types impinging on a sample of pure nickel at $1 \mathrm{MeV}$. The displacement efficiency is defined as the fraction of the "ballistically" produced Frenkel pairs (FPs), which survive the cascade quench and are available for long-range migration. These are referred to variously as "freely migrating" [13] or "available migrating" [14] defects. They are the only defects that will affect the amount of grain boundary segregation, our measure of radiation effect. The fraction of the total number of defects produced that are "freely migrating" is termed the displacement efficiency, $\varepsilon$. Despite the equivalence in energy among the four particle types, the average energy transferred and the defect production efficiencies vary by almost two orders of magnitude! This is explained by the differences in the cascade morphology among the different particle types. Neutrons and heavy ions produce dense cascades that result in substantial recombination during the cooling or quenching phase. However, electrons are just capable of producing a few widely spaced Frenkel pairs that have a low probability of recombination. Protons produce small widely spaced cascades and many isolated FPs due to the Coulomb interaction and, therefore, fall between the extremes in displacement efficiency defined by electrons and neutrons.

We will focus on the comparison between four types of particle irradiation in order to outline a methodology for establishing equivalence between neutron and charged particle irradiation. The four types are given in Table 1 and are taken from experiments conducted to study the IASCC problem [1518]. Each experiment is characterized by the particle type and energy, irradiation temperature, reported dose rate, and reported total dose. The displacement efficiency is calculated using Naundorf's model [19], which is based on two factors. The first is that the energy transfer to atoms is only sufficient to create a single Frenkel pair. The second is that the Frenkel pair lie outside a recombination (interaction) radius so that the nearby FPs neither recombine nor cluster.
The model follows each generation of the collision and calculates the fraction of all defects produced that remain free. According to Naundorf, the free single FPs are classified according to the generation $i$ in which they were produced, that is, the relative amount $\eta_{1}$ is the amount that is produced by primary collisions (first generation), while $\eta_{2}$ is the relative amount produced by secondary collisions (second generation). Thus, the total number of free single FPs produced is:

$\eta=\sum_{i} \eta_{i}$

where that produced by primary collisions is:

$\eta_{1}=\left(\beta_{p} / \sigma_{d}\right) \int_{E_{d}}^{\alpha E} d T K_{I, A}(E, T)$,

and that produced by secondary collisions is:

$$
\begin{gathered}
\eta_{2}=\left(1 / \sigma_{d}\right) \int_{E_{d}}^{a E} d T K_{I, A}(E, T)\left[Z(T) \beta_{A}(T) / \sigma_{A}(T)\right] \\
\int_{E_{d}}^{2.5 E_{d}} d T^{\prime} K_{A, A}\left(T, T^{\prime}\right) .
\end{gathered}
$$

The primary displacement cross section for the incident ion is:

$\sigma_{p}=\int_{E_{d}}^{\alpha E} d T K_{I, A}(E, T)$,

and the total displacement cross section $\sigma_{d}$ is given in the Kinchen-Pease model by:

$\sigma_{d}=\int_{E_{d}}^{\alpha E} d T K_{I, A}(E, T) v(T)$.

$K_{I, A}(E, T)$ is defined as the differential cross section of an incident ion $(I)$ of energy $E$, which transfers the energy $T \leqslant T_{\max }$ to an atom (A) of the crystal, $E_{d}$ is the displacement energy, the maximum energy transferred is $\alpha E\left(\alpha=4 M_{I} M_{A} /\left(M_{I}+M_{A}\right)^{2}\right)$ and $v(T)$ is the $K-P$ displacement function defined earlier. $Z(T)$ is the total number of secondary collisions produced above $E_{d}$ by a primary of energy $T$ along its path. The distance $\lambda$ between two primary collisions is distributed according to an exponential law,

$W(\lambda)=\left(1 / \lambda_{p}\right) \exp \left(-\lambda / \lambda_{p}\right)$, 
Table 1 Comparison of Different Particle Irradiation Experiments

\begin{tabular}{|c|c|c|c|c|c|c|c|}
\hline \multirow{2}{*}{$\begin{array}{l}\text { Particle } \\
\text { type }\end{array}$} & \multirow{2}{*}{$\begin{array}{c}\text { Energy } \\
\mathrm{MeV}\end{array}$} & \multirow{2}{*}{$\begin{array}{l}\text { Irradiatior } \\
\text { temper- } \\
\text { ature } \\
\left({ }^{\circ} \mathrm{C}\right)\end{array}$} & \multirow[b]{2}{*}{ Efficiency } & \multicolumn{2}{|c|}{$\begin{array}{l}\text { Displacement rate } \\
\quad(\text { dpa/s) }\end{array}$} & \multicolumn{2}{|c|}{$\begin{array}{l}\text { Total dose } \\
\quad(\text { dpa })\end{array}$} \\
\hline & & & & Reported & Real & Reported & Real \\
\hline \multicolumn{8}{|l|}{ Electrons } \\
\hline Kato [15] & 1.0 & 450 & 1.0 & $1.9 \times 10^{-3}$ & $1.9 \times 10^{-3}$ & 10 & 10 \\
\hline \multicolumn{8}{|l|}{ Protons } \\
\hline Was [16] & 3.4 & 400 & 0.2 & $7.0 \times 10^{-6}$ & $1.4 \times 10^{-6}$ & 1 & 0.2 \\
\hline $\mathrm{Ni}^{++}$ions & & & & & & & \\
\hline Bruemmer [17] & 5.0 & 500 & 0.04 & $5.0 \times 10^{-3}$ & $2.0 \times 10^{-4}$ & 10 & 0.4 \\
\hline $\begin{array}{l}\text { Neutrons } \\
\text { Jacobs [18] }\end{array}$ & $\begin{array}{l}\text { Fission } \\
\text { reactor }\end{array}$ & 288 & 0.02 & $\begin{array}{c}\sim 4.5 \times 10^{-8} \mathrm{dpa} / \mathrm{s} \\
1 \times 10^{21} \mathrm{n} / \mathrm{cm}^{2} \\
(E>1 \mathrm{MeV})\end{array}$ & $9.0 \times 10^{-10}$ & 1 & 0.02 \\
\hline
\end{tabular}

with the mean distance:

$\lambda_{p}=\Omega / \sigma_{p}$,

where $\Omega$ is the atomic volume. The condition that the distance between two consecutive collisions must be larger than an appropriate interaction radius, $r_{i v}$ (so that FPs produced near each other neither recombine nor cluster) reduces the amount of all possible free single FPs by:

$\beta_{p}=\exp \left(-r_{i v} / \lambda_{p}\right)$.

Results of the model are the displacement efficiencies shown for the four particle types in Table 1. The "corrected" displacement rate and "corrected" total dose for each particle type are determined by multiplying the reported (uncorrected) values times the efficiency factor.

\section{MEASURING RADIATION-INDUCED SEGREGATION}

Radiation-induced segregation near grain boundaries is measured using two principle methods-energy-dispersive X-ray analysis in STEM-EDS and AES. In STEM-EDS, the electron beam is focused to a spot on the order of $2 \mathrm{~nm}$ diameter and used as a probe for the generation of $X$-rays from the volume of the foil through which it passes. The X-ray intensities can be used to determine the com- position of the material in the sample volume. The composition profile of an element near a grain boundary is then determined by stepping the electron beam probe across the grain boundary and converting the $X$ ray signal intensity profile to a composition profile. STEM-EDS is a tricky technique that requires a fine probe size $(\leqslant 2 \mathrm{~nm})$, a grain boundary that can be accurately aligned with the probe direction, and a sampling region in which the foil thickness is less than $100 \mathrm{~nm}$ and is accurately known. Even so, the finite volume of the electron beam probe and the broadening of the initial probe size inside the sample due to electron scattering limit the spatial resolution of the technique to 2-3nm at best.

An alternative technique is AES, which involves the identification and quantitative determination of elements from the energy of the Auger electrons emitted from the interaction of an electron beam with a surface. This technique requires a different sample geometry. In AES, the composition of a surface is determined by directing the electron beam normal to that surface. As such, in order to investigate grain boundary compositions, the sample must be fractured along the grain boundaries. This is usually accomplished in stainless steels by cathodic charging with hydrogen and straining in situ in the Auger vacuum chamber. As such, unless 
Table 1 (Continued)

\begin{tabular}{|c|c|c|c|c|c|}
\hline \multicolumn{2}{|c|}{$\begin{array}{c}\text { Amount of } \mathrm{Cr} \text { depl. }(\dot{A}), \\
\text { based on: }\end{array}$} & \multicolumn{2}{|c|}{$\begin{array}{l}\text { Dose to reach steady } \\
\text { state (dpa), based on: }\end{array}$} & \multicolumn{2}{|c|}{$\begin{array}{l}\text { Amount of } \mathrm{Cr} \text { depl. (A) } \\
\text { at steady state, based on: }\end{array}$} \\
\hline $\begin{array}{l}\text { Reported } \\
\text { dose rate }\end{array}$ & $\begin{array}{c}\text { Real } \\
\text { dose rate }\end{array}$ & $\begin{array}{l}\text { Reported } \\
\text { dose rate }\end{array}$ & $\begin{array}{c}\text { Real } \\
\text { dose rate }\end{array}$ & $\begin{array}{l}\text { Reported } \\
\text { dose rate }\end{array}$ & $\begin{array}{c}\text { Real } \\
\text { dose rate }\end{array}$ \\
\hline 4.4 & 4.4 & 28 & 28 & 5.2 & 5.2 \\
\hline 7.1 & 5.4 & 7 & 3 & 9.7 & 11.4 \\
\hline 4.9 & 4.8 & 25 & 7 & 5.6 & 9.1 \\
\hline 8.7 & 2.4 & 4 & 1.4 & 10.9 & 14.3 \\
\hline
\end{tabular}

sputter depth profiling can be accomplished on a faceted surface (generally very difficult), the information on sample composition is limited to the grain boundary plane itself. In AES, the depth resolution rather than the spatial resolution is a concern. The nominally $10 \mathrm{keV}$ electrons penetrate and produce Auger electrons over several nanometers below the surface. However, those collected by the cylindrical mirror analyzer only come from a few angstroms corresponding to the escape depth of the Auger electrons. Therefore, the accuracy of the measurement is a function of the escape depth of the Auger electrons of interest. Additional practical considerations influence measurements, such as the contamination of the surface with $C$ and $O$ from the vacuum, the uncertainty in the location of the fracture along the grain boundary plane, and the possibility of erring on identification of grain boundary facets. These two techniques provide all the information we have on RIS at grain boundaries.

\section{RESULTS AND DISCUSSION}

\section{MODEL RESULTS}

As a means of comparing the expected measured amount of grain boundary segregation for each of the irradiations described in
Table 1, we used the Lam model [8] to simulate the depletion of chromium at the grain boundary of 304 stainless steel of composition Fe-20Cr-9Ni. Numerical solutions are made for a sample under uniform irradiation with energetic particles assuming a spatially uniform production rate of defects for each particle type. Particle type is identified by the input displacement rate. Model calculations are made by using the same assumptions and boundary conditions and material parameters as given by Lam et al. [8] Vacancies were assumed to migrate via the alloying elements with a migration energy of $1.3 \mathrm{eV}$, which is the same as the energy for vacancy migration in pure Fe. However, according to Lam, from the different values of $D_{o}$ measured experimentally (see Lam et al. [8]), the preexponential attemptfrequency factors for $\mathrm{Ni}, \mathrm{Cr}$, and $\mathrm{Fe}$ were different. No preferential association of defects with $\mathrm{Fe}, \mathrm{Cr}$, or $\mathrm{Ni}$ atoms is assumed. Segregation of the alloying elements results from the difference in vacancy diffusion coefficients caused only by the inequality of the preexponential jump-frequency factors. There is no effect of interstitial fluxes because all the partial diffusion coefficients of interstitials via the three alloying elements are equal.

Results show that enrichment of $\mathrm{Ni}$ and depletion of $\mathrm{Cr}$ and Fe occur in agreement with the calculations of Lam et al. [8, 20]. 
The quantity of interest is the amount of chromium depleted from the grain boundary, or the area inside the $\mathrm{Cr}$ concentration profile (Fig. 3). The appropriate measure of depletion is somewhat questionable. One could use the grain boundary chromium value as the measure of the extent of chromium depletion. Alternatively, the full width at half maximum (FWHM) of the depletion profile has been used. In fact, both of these quantities are useful and can be obtained from measured depletion profiles. However, the area inside the $\mathrm{Cr}$ concentration profile represents changes to a volume of material and, as will become evident later, is more sensitive to changes in the profile shape than either the grain boundary value or the FWHM alone. The amount of $\mathrm{Cr}$ depletion is determined by integrating the concentration profile for that element with distance from the grain boundary,

$M=\int_{0}^{l(t)}\left[C_{A}^{o}-C_{A}(x, t)\right] d x$,

where $M$ is the segregated area, $C_{A}^{0}$ is the bulk atom concentration, $C_{A}(x, t)$ is the atom concentration near the surface, and $l(t)$ is the half-width of the depleted zone. The amount of $\mathrm{Cr}$ depletion for "corrected" and "reported" dose rates is given in Table 1 for each experiment. Also given are the values of $\mathrm{Cr}$ depletion at steady state and the doses

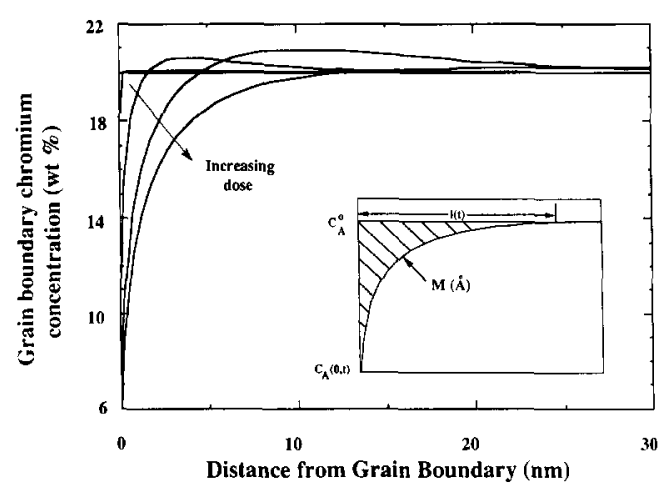

FIG. 3. Chromium concentration profile near the grain boundary in Fe-20Cr-9Ni during particle bombardment at a displacement rate of $10^{-3}$ at $500^{\circ} \mathrm{C}$. The inset defines the variables used to describe the amount of Cr depletion, $M$, defined in the text.

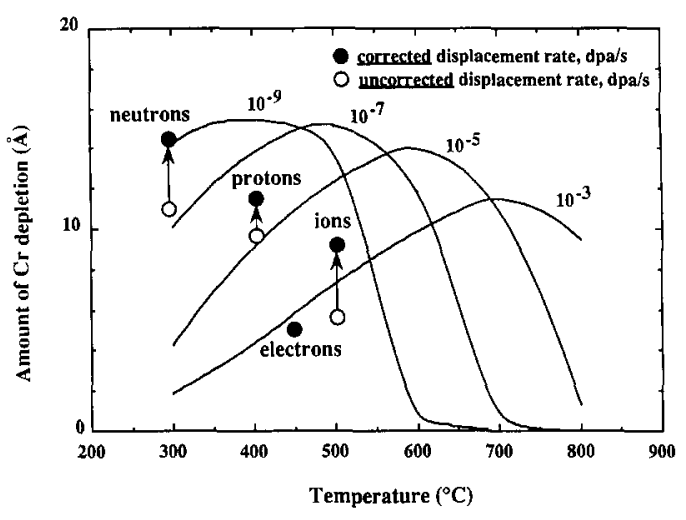

Fig. 4. Comparison of the amount of chromium depletion at steady state, as a function of temperature, dose rate, and displacement efficiency for each particle type. The symbols represent the reported (uncorrected), and efficiency-corrected dose rates for each particle type under the steady state conditions reported in Table 1.

required to reach steady state. Figure 4 shows the amount of $\mathrm{Cr}$ depletion as a function of temperature and displacement rate at steady state. Steady state is reached at different dose levels for each experiment. At a given displacement rate, the segregated area peaks at some intermediate temperature and falls off at both higher and lower temperatures. This is due to the dominance of recombination at low temperatures and back diffusion at high temperatures [21]. Also, note that the effect of a decreasing displacement rate is to shift the curves to higher maxima at lower temperatures. For a given dose, a lower displacement rate yields lower steady-state defect concentrations, reducing the number of defects lost to recombination, increasing the peak and shifting it to lower temperatures.

Note the change in the calculated values for the amount of $\mathrm{Cr}$ depletion in the four experiments shown in Fig. 4. Because electrons are assumed to be $100 \%$ efficient in producing defects available to affect segregation, there is no change in the segregated area after accounting for efficiency. However, there is a difference with protons, heavy ions, and neutrons. The difference is largest for neutrons and smallest for protons. The difference is a function of not only the displacement efficiency, but also the slope of the dose rate curves. Nevertheless, 
substantial differences result in the expected amounts of grain boundary segregation when the displacement efficiency is taken into account.

Figure 4 shows the effect of three of the four parameters defining an experiment: particle type, temperature, and dose rate. It does not show the effect of dose because this is a steady-state result that is achieved at different doses for each of the experiments described in Table 1. Figure 5 shows the dose required to reach steady state as function of temperature and dose rate. Each of the experiments are plotted for both the reported and the corrected displacement rates. Note the large difference in the dose to reach steady state between electrons and neutrons. In general, irradiation at a lower dpa rate will result in a lower dose at steady state, and the difference is greatest for this comparison. Correspondingly, proton and heavy ion irradiation fall between neutrons and electrons for the experiments described in Table 1.

The series of curves in Fig. 6(a,b) show the development of the segregated area due to chromium depletion (versus dose and irradiation time, respectively) during irradiation for each of the four experiments described in Table 1. Each particle type generates a pair of curves, the top curve (at large dose) re-

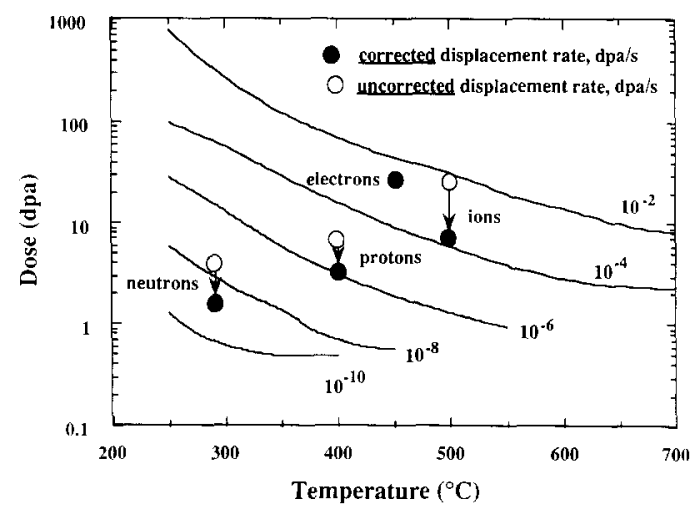

FIG. 5. Dose required to reach steady state as a function of temperature, dose rate, and particle efficiency for each particle type. The symbols represent the reported (uncorrected), and efficiency-corrected dose rates for each particle type under the steady-state conditions reported in Table 1 .

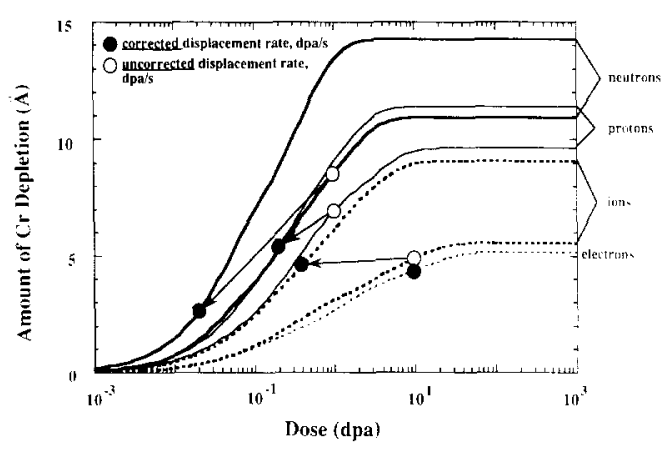

(a)

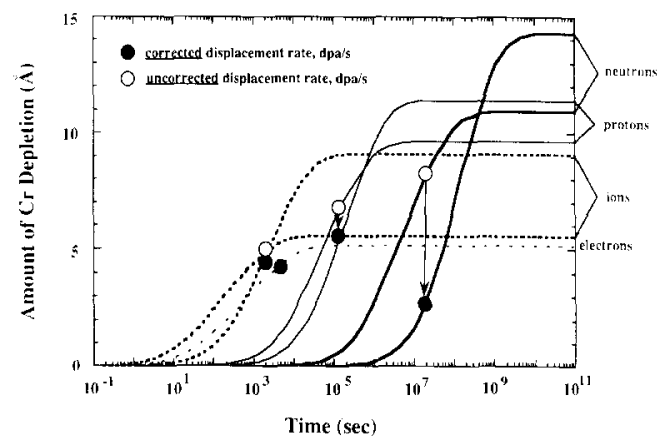

(b)

Fig. 6. Amount of chromium depletion as a function of irradiation dose (a) and time (b) for each particle type. The upper curve (at steady state) in each pair accounts for the particle efficiency, while the lower curve is for the reported (uncorrected) dose rate. The symbols refer to the conditions for the experiments described in Table 1.

sults from use of the corrected displacement rate and the bottom curve from use of the reported displacement rate. A single curve was used for electron irradiation because the efficiency of defect production for electrons was assumed to be $100 \%$. The points on the curves indicate the doses (times) to which these experiments were conducted. The arrows indicate the changes in the measured depletion when the reported displacement rate and dose are corrected using the efficiency factors in Table 1. In none of the experiments is steady state reached after the reported dose. This is particularly true for electron irradiation where even after 10dpa, the steady-state dose is still a factor of 3 away. Even in the case of neutrons, a real dose of 1dpa (just past the threshold for observation 
of IASCC) falls on the steep portion of the curve, indicating that the measured amount of segregation is very sensitive to the duration of the experiment.

A final point of importance is the amount of $\mathrm{Cr}$ depletion that would be measured for each of the particle types after the stated duration of each experiment. Figure 6(a) shows that for these four experiments, the amount can vary by almost a factor of 3 between various particle types. This is essentially an effect of the rate dependence on the amount of segregation. The curves in Fig. 6(a) also show the interplay between rate and efficiency for a fixed amount of segregation. Low efficiencies or low displacement rates will produce greater amounts of segregation after a fixed dose than higher efficiencies or higher displacement rates. This requires trade-offs in experiments using ion irradiation to study neutron damage. Generally, the efficiency is better for lighter ions, and due to the nature of the instrumentation (accelerators, HVEM microscope), damage rates are generally much higher.

Figure 7 summarizes the resulting discrepancies between determination of the amount of segregation using the "reported" dpa rates and doses versus "corrected" values that account for the efficiency of producing freely migrating defects available for affecting segregation. As expected, the difference is generally largest for the particle type with the greatest correction to the dpa rate. However, the difference is strongly dependent on the dose to which the experiment is carried. In fact, ion irradiation experiments near 10dpa (0.4 "real" dpa) will show virtually no discrepancy due to the crossover of the segregation curves at that dose.

Although steady state represents a convenient point in the dose history with which to evaluate the effect of particle efficiency, few LWR components or ion irradiation experiments are taken to steady state. In particular, the dose regime of interest in the practical problem is $0.5-5 \mathrm{dpa}$. As such, Fig. 8 shows how the grain boundary chromium concentration varies with particle type (using corrected dose rates) for the relevant tem-

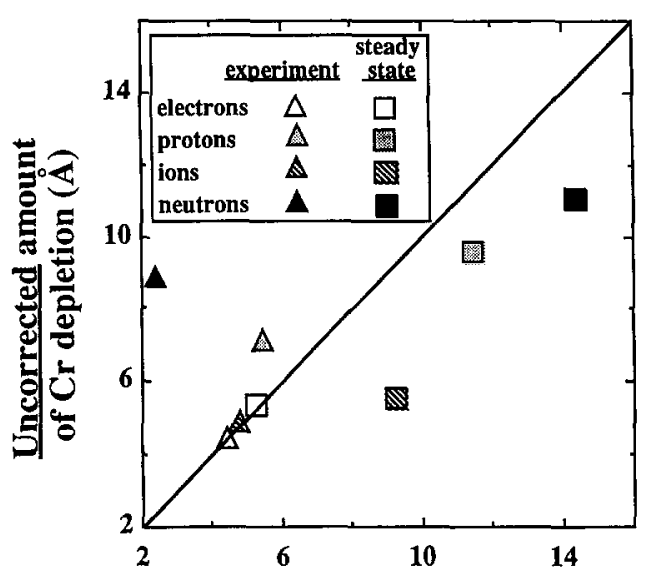

Corrected amount of $\mathrm{Cr}$ depletion (̊)

Fig. 7. Comparison of the amount of chromium depletion accounting for particle efficiency (corrected amount of $\mathrm{Cr}$ depletion) and that using the reported (uncorrected) values of dose and dose rate (uncorrected amount of $\mathrm{Cr}$ depletion) for the experiments given in Table 1 and at steady state.

peratures at a dose of $1 \mathrm{dpa}$. Note that there is a significant shift in the minimum to lower temperatures with decreasing dose rate. The effect of accounting for particle efficiency will be greatest for neutrons $(\varepsilon=0.02)$ and is shown in the plot of grain boundary $\mathrm{Cr}$ concentration versus temperature in Fig. 9. Here, the temperature at which the minimum grain boundary $\mathrm{Cr}$ concentration is reached is shifted by almost $100^{\circ} \mathrm{C}$ ! This figure illus-

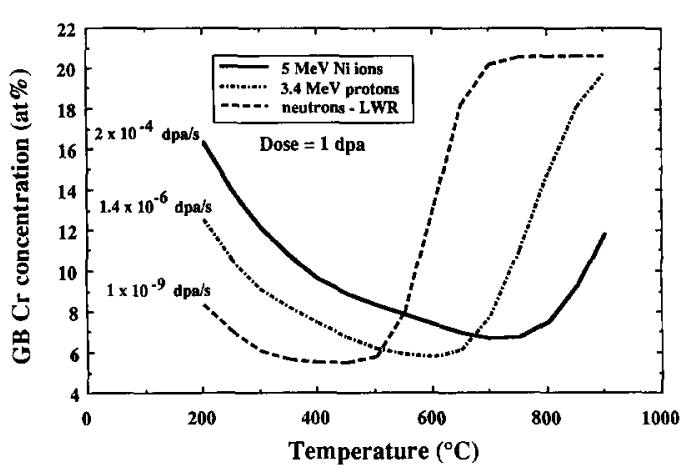

Fig. 8. Effect of particle type on the grain boundary chromium concentration in stainless steel at 1dpa as a function of temperature. The dose rates correspond to the corrected values used in the experiments given in Table 1. 


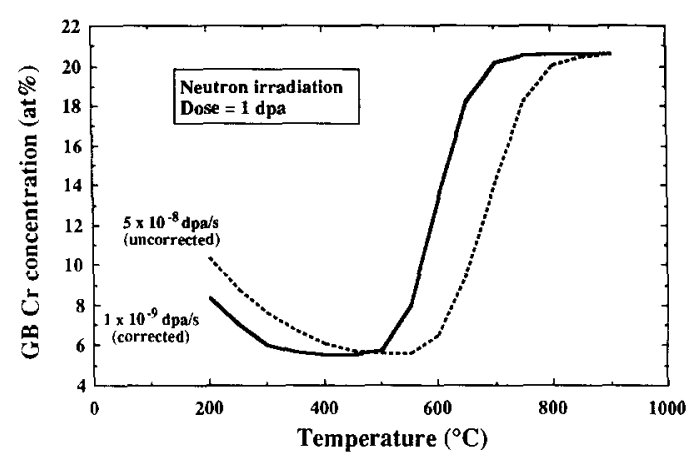

FIG. 9. Effect of displacement efficiency on the grain boundary chromium concentration in stainless steel as a function of temperature during irradiation with neutrons to $1 \mathrm{dpa}$.

trates the consequences of not accounting for particle type in the modeling of radiationinduced segregation.

These examples also illustrate the methodology for determining equivalence between grain boundary composition for irradiation with different particle types. In practice, the method may be used either to determine the irradiation conditions that achieve equivalence a priori, or to determine the relative amounts of grain boundary segregation "expected" given the parameters defining the experiment.

\section{COMPARISON WITH EXPERIMENTS}

For comparison with experiment, the "Perks" computer model [10] (as constructed by Simonen [11]) was used to make model calculations. For the purposes of these calculations, it is essentially the same model as that written by Lam [8]. In fact, cases run with both codes using the same parameter set produce nearly identical values for element concentration profiles at grain boundaries. Chromium concentration profiles at the grain boundaries are shown in Figs. 1012, which compare the measured data taken by STEM and AES to the model calculations for several irradiations. Figure 10 presents results from proton irradiation, Fig. 11 from neutron irradiations and Fig. 12 from heavy-ion irradiations. The irradiation conditions for protons and ions are identical to

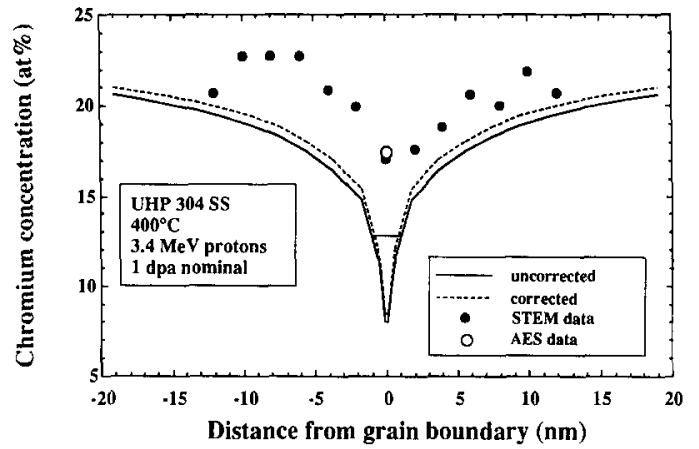

(a)

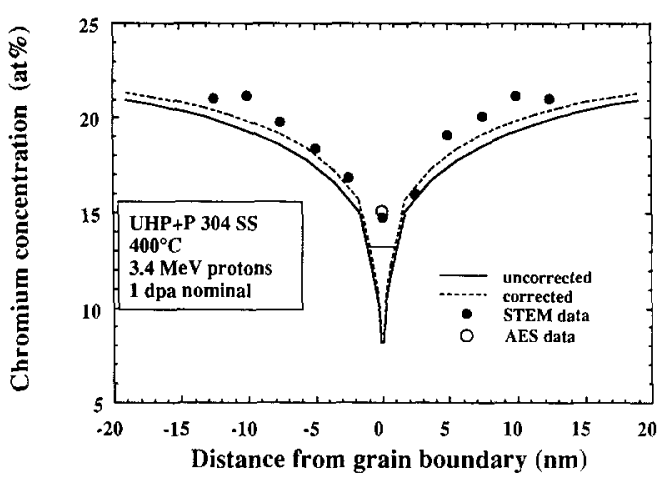

(b)

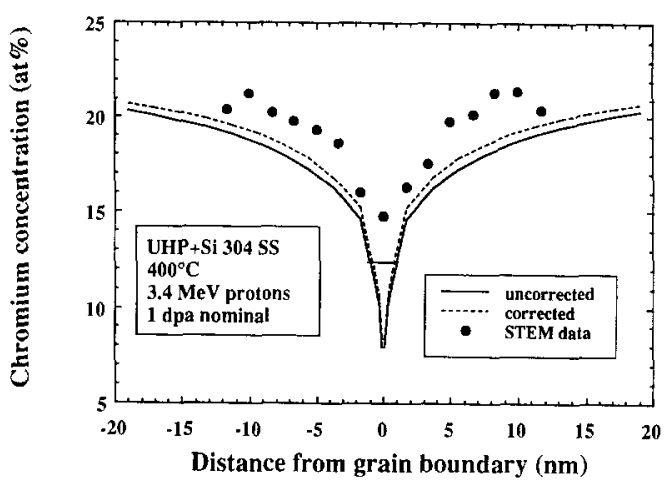

(c)

FIG. 10. Comparison of experiment and model results of the chromium depletion profile in stainless steel following irradiation with $3.4 \mathrm{MeV}$ protons at $400^{\circ} \mathrm{C}$ to $1 \mathrm{dpa}$, (a) UHP alloy, (b) UHP + P alloy, and (c) UHP + Si alloy. The horizontal bar represents the diameter of the incident electron probe in STEM-EDS measurements.

those in Table 1 with the exception of the final dose of the ion irradiation. The neutron irradiations were all done at $288^{\circ} \mathrm{C}$, but doses and dose rates vary according to the 


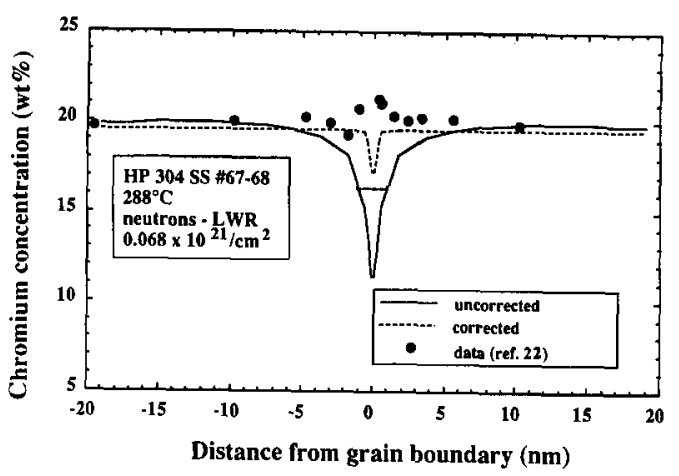

(a)

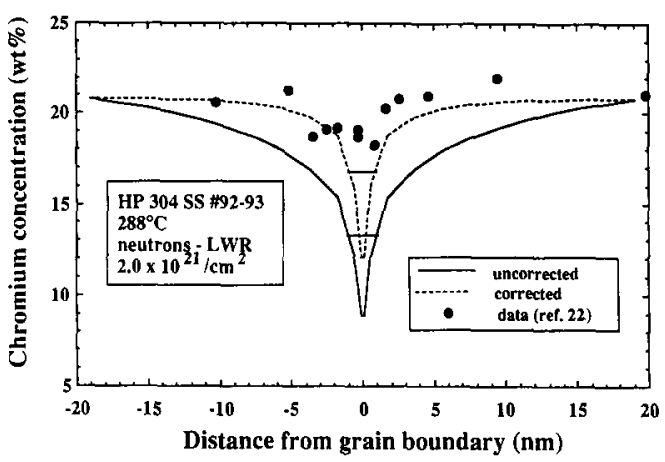

(b)

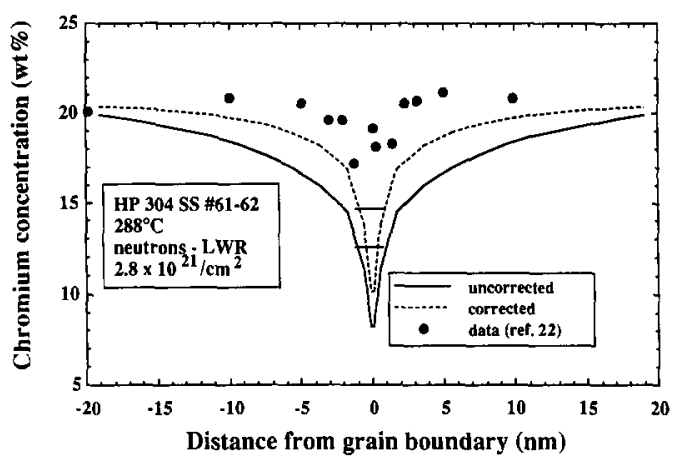

(c)

reactor in which they were done. Neutron irradiations were uncontrolled in that the reported grain boundary composition profiles were taken from core components and not from controlled environment test capsules. Samples in Fig. 11(a-d) were from a lightwater reactor [22] and that from Fig. 11(e) is from the advanced test reactor (ATR) [23]. Each graph shows the experimental data, the code prediction for the nominal condi-

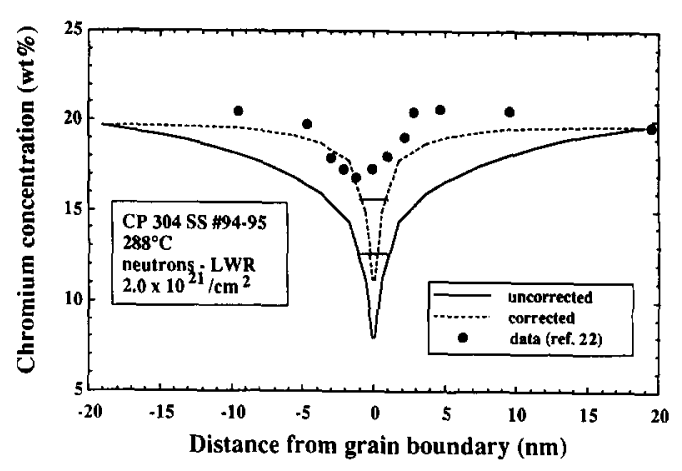

(d)

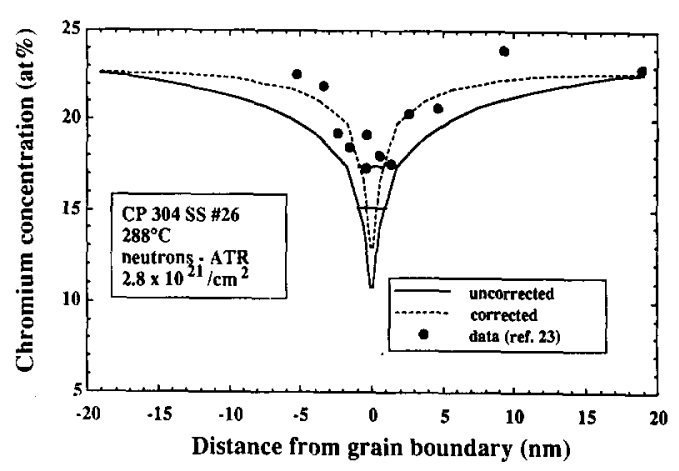

(e)

FIG. 11. Comparison of experiment and model results of the chromium depletion profile in stainless steel following neutron irradiation at $288^{\circ} \mathrm{C}$ at various doses with $E \geqslant 1 \mathrm{MeV}$ (a) HP alloy, $0.068 \times 10^{21} \mathrm{n} / \mathrm{cm}^{2}$ in LWR (b) HP alloy, $2.0 \times 10^{21} \mathrm{n} / \mathrm{cm}^{2}$ in LWR, (c) HP alloy, 2.8 $\times 10^{21} \mathrm{n} / \mathrm{cm}^{2}$ in LWR, (d) CP alloy, $2.8 \times 10^{21} \mathrm{n} / \mathrm{cm}^{2}$ in LWR, and (e) CP alloy, $2.8 \times 10^{21} \mathrm{n} / \mathrm{cm}^{2}$ in ATR. The horizontal bar represents the diameter of the incident electron probe in STEM-EDS measurements.

tions, and that accounting for the particle efficiency.

Figure 10(a-c) shows the comparison of STEM-EDS measurements and code calculations for three controlled-purity alloys irradiated with $3.4 \mathrm{MeV}$ protons at $400^{\circ} \mathrm{C}$ to $1 \mathrm{dpa}$ (nominal) [24]. The dotted line is the "corrected" $\mathrm{Cr}$ profile determined using the efficiency-corrected dose rate, and the solid line is the uncorrected profile. Figure 10(a) 


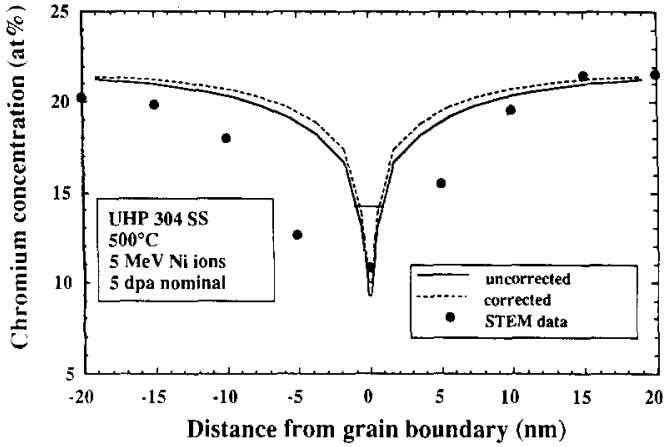

Fig. 12. Comparison of experimental and model results of the chromium depletion profile in the UHP alloy following irradiation with $5 \mathrm{MeV} \mathrm{Ni}^{++}$at $500^{\circ} \mathrm{C}$ to $5 \mathrm{dpa}$. The horizontal bar represents the diameter of the incident electron probe in STEM-EDS measurements.

for the high-purity alloy shows reasonably good agreement between code and experiment in shape, but not in magnitude. From both the grain boundary value and the FWHM of the profile, it is evident that the code calculations predict a greater degree of chromium depletion. Consistency between experimental techniques is indicated by the excellent agreement between STEMEDS and AES on the value of $\mathrm{Cr}$ at the grain boundary. Figure $10(b, c)$ shows that the calculated profiles match the experimental profiles much better in the UHP $+\mathrm{P}$ and $\mathrm{UHP}+\mathrm{Si}$ alloys. These alloys are essentially the same as the UHP alloy in Fig. 10(a) except that they are doped with $\mathrm{P}$ and $\mathrm{Si}$, respectively. In both cases, the shape and magnitude of the experimental and calculated results match very well beyond $2 \mathrm{~nm}$ from the grain boundary. The lack of agreement within $2 \mathrm{~nm}$ of the grain boundary is a consistent problem. In part, it may be due to the lack of spatial resolution in STEM-EDS and depth resolution in AES. The better agreement between experiment and theory for the doped alloys is not unexpected. Evidence exists to show that the addition of $P$ and $\mathrm{Si}$ to $\mathrm{Fe}-\mathrm{Cr}-\mathrm{Ni}$ increases the effective vacancy diffusion coefficient, thus leading to a broader and deeper profile under irradiation [25]. An additional factor that may affect the grain boundary value is the in- crease in the vacancy formation energy by the addition of solutes that decrease the equilibrium vacancy concentration at the boundary and result in an increased vacancy flux to the boundary [26].

Figure 11(a-c) shows STEM-EDS results compared with code calculations for a highpurity alloy irradiated to increasing doses. Figure 11(d, e) shows the comparison for commercial-purity alloys: the former irradiated in an LWR and the latter in a test reactor (ATR). Code calculations were renormalized to match the measured chromium levels at $20 \mathrm{~nm}$ from the grain boundary. Inspection of Fig. 11 shows that the code significantly underpredicts the grain boundary composition in all the neutron irradiated cases. This is in agreement with results of Norris et al. [27] using Perks' model [10] in a comparison with neutron irradiated $20 \% \mathrm{Cr} / 25 \% \mathrm{Ni} / \mathrm{Nb}$ stabilized steel used as CAGR fuel pin cladding. It also shows that the effect of the particle efficiency can be very significant. In particular, except for the profile being off center, Fig. 11(d) shows rather good agreement between experiment and corrected profile beyond $2 \mathrm{~nm}$ from the grain boundary. Similarly, the ATR data [Fig. 11(e)] also shows better agreement beyond $2 \mathrm{~nm}$. If one considers the purity of these samples, the results support those found in the proton-irradiated UHP alloys, showing that impurities increase the amount of $\mathrm{Cr}$ segregation at the grain boundary.

Figure 12 shows the comparison between model and experiment for the UHP alloy of Fig. 10(a) irradiated with $5 \mathrm{MeV} \mathrm{Ni}^{++}$ions to a dose of $5 \mathrm{dpa}$ at $500^{\circ} \mathrm{C}$. This result is somewhat peculiar in that it is the only case in which the experimental measurements produce a broader profile than the model.

\section{PROBLEMS AND INCONSISTENCIES}

\section{Experiment}

Various problems can be identified with the experimental data used for this analysis. The first involves the knowledge of the true dpa and dpa rate for comparison with the model. 
Although an attempt was made to rectify this problem using calculated efficiencies, a better understanding of point defect behavior in an irradiated structure is needed in order to determine the true damage rate. Further, close control of the irradiation environment in experiments is required to produce more accurate dose and dose rate values.

A second area of concern is the difficulty in accurately determining the composition right on the grain boundary either by AES or STEM-EDS. Both methods have shortcomings that may not be resolvable due to the finite nature of the electron probe in STEM and the depth penetration in AES. From this standpoint, it may be more prudent to use the width of the depleted zone as a measure of the degree of segregation. The results presented in Figs. 3-7 used the depletion volume, which is probably the best measure of the extent of depletion. However, this is rather arbitrarily defined, while the FWHM is a more readily determinable quantity.

\section{Computational Models}

The paramount drawback with the models presently available is the lack of data to determine accurately the input parameters, such as formation and migration energies and jump frequencies. Variations in these values by only small percentages can cause large changes in the resulting grain boundary composition profiles. Other areas of concern are the effect of impurities and sinks. It should be noted that irradiation to the same dose results in greater segregation in the commercial-purity material than the high-purity material. This is also true in the proton-irradiated alloys, where the $\mathrm{P}$ - and Si-doped alloys show greater segregation (and better agreement with the model results) than the UHP alloy. In addition to impurities, the effect of point defect sinks may be of concern. At high sink densities, the increased removal of point defects leads to a shallower and more narrow depletion profile. The dynamic evolution of microstructure is not accounted for in any RIS code but may only become significant at very high sink levels, corresponding to very high doses.

\section{SUMMARY}

The efficiency of producing "freely migrating" defects that are available for affecting processes such as segregation can have a large impact on the resulting degree of segregation. Efficiencies range from 1 for $\mathrm{MeV}$ electrons to 0.02 for neutrons with $E \geqslant 1 \mathrm{MeV}$. Lower efficiencies translate into a lower displacement rate, changing the degree of grain boundary segregation and the temperature dependence. Experiments that use charged particles to study neutron damage generally have higher displacement efficiencies and are conducted at higher displacement rates, requiring larger nominal doses to achieve the same measure of radiation effect. The dose to which experiments are conducted is also very important in comparing segregation between various particle types. Because most experiments do not reach steady state, the amount of segregation is a sensitive function of the total dose. Hence, comparisons between particle types can yield fortuitous agreement or disagreement depending on the length of the experiment. Comparison between model and experimental results on chromium redistribution in stainless steels irradiated with neutrons, protons, and heavy ions shows that, generally, the measured depletion profile is narrower and shallower than that calculated from theory. The origins of this difference are probably due to spatial and depth resolution of the STEM-EDS and AES measurement techniques, respectively, the lack of knowledge of the material parameters in modeling and the uncertainty in the true displacement rate. Nevertheless, beyond $2 \mathrm{~nm}$ from the grain boundary, the shape of the experimental and calculated profiles agree reasonably well.

The authors gratefully acknowledge the National Science Foundation for partial financial support of T. Allen. Partial support for this work was also provided by the U.S. Department of Energy, 
grant no. DE-FG07-88ER12825. Partial support from the General Electric Company is also acknowledged.

\section{References}

1. G. S. Was and P. L. Andresen, JOM 44:8 (1992).

2. P. L. Andresen, F. P. Ford, S. M. Murphy, and J. M. Perks, Proc. Fourth International Symposium on Environmental Degradation of Materials in $\mathrm{Nu}$ clear Power Systems - Water Reactors, National Association of Corrosion Engineers, Houston, TX, pp. 1-83 (1990).

3. D. C. Bullington, Yankee Irradiation Program, Summary Report, part I. Yankee In-Pile Irradiation Test, USAEC report YAEC-182, Westinghouse Elec. Corp. (1962).

4. J. B. Brown, V. W. Storhok, and J. E. Gates, Trans. Am. Nucl. Soc. 10:668 (1967).

5. C. F. Cheng, Corrosion 20:341 (1964).

6. V. Pasupathi and R. W. Klingensmith, Investigation of Fuel Rod Failures in the Connecticut Yankee Reactor, BCL-585-19, Nov. (1980).

7. P. R. Okamoto and L. E. Rehn, J. Nucl. Mat. 83:2 (1979)

8. N. Q. Lam, A. Kumar, and H. Wiedersich, in Effects of Radiation on Materials: Eleventh Conference, ASTM STP782, H. R. Brager and J. S. Perrin, eds., American Society for Testing and Materials, 1982, p. 985.

9. A. C. Hindmarsh, Lawrence Livermore Laboratory Report UCID-30002, 1974.

10. J. M. Perks, A. D. Marwick, and C. A. English, Proc. Radiation-Induced Sensitisation of Stainless Steels, D. I. Norris, ed., Central Electricity Generating Board, Berkeley Nuclear Laboratories, Berkeley, Gloucestershire, p. 15 (1987).

11. E. Simonen, Private Communication (April 1992).

12. G. H. Kinchen and R. S. Pease, Rep. Prog. Phys. 18:1 (1955).

13. L. E. Rehn, J. Nucl. Mat. 174:144 (1990).
14. L. K. Mansur and K. Farrell, J. Nucl. Mat. 170:236 (1990).

15. T. Kato, H. Takahashi, S. Ohnuki, K. Nakata, and J. Kuniya, J. Nucl. Mat. 623:179-181 (1991).

16. D. Damcott, D. Carter, J. Cookson, J. Martin, M. Atzmon, and G. S. Was, Rad. Eff. and Def. in Solids 118:383 (1991).

17. S. Bruemmer, J. Nucl. Mat. 186:13 (1991).

18. A. J. Jacobs and G. P. Wozadlo, Corrosion 91, paper \#41, National Association of Corrosion Engineers, Houston, TX (1991).

19. V. Naundorf, J. Nucl. Mat. 182:254 (1991).

20. V. K. Sethi and P. R. Okamoto, in Phase Stability During Irradiation, J. R. Holland, L. K. Mansur and D. I. Potter, eds., p. 109, The Metallurgical Society of AIME, Warrendale, PA (1981).

21. P. R. Okamoto and L. E. Rehn, J. Nucl. Mat. 83:2 (1979).

22. A. J. Jacobs, Effects of Radiation on Materials: 16th International Symposium, ASTM STP 1175, A. S. Kumar, D. S. Gelles, R. K. Nanstad, and E. A. Little, eds., American Society for Testing and Materials, Philadelphia, p. 902 (1993).

23. A. J. Jacobs, G. E. C. Bell, C. M. Shepherd, and G. P. Wozadlo, Proc. Fifth International Symposium on Environmental Degradation of Materials in $\mathrm{Nu}$ clear Power Systems-Water Reactors, D. Cubicciotti, ed., American Nuclear Society, La Grange Park, IL, p. 917 (1992).

24. R. D. Carter, D. L. Darncott, M. Atzmon, G. S. Was, and E. A. Kenik, J. Nucl. Mat. 205:361 (1993).

25. F. A. Garner and H. R. Brager, J. Nucl. Mat. 133 \& 134:511 (1985).

26. A. Azarian and K. Kheloufi, J. Nucl. Mat. 97:25 (1981).

27. D. I. R. Norris, C. Baker, and J. M. Titchmarsh, Proc. Radiation-Induced Sensitisation of Stainless Steels, D. I. Norris, ed., Central Electricity Generating Board, Berkeley Nuclear Laboratories, Berkeley, Gloucestershire, p. 86 (1987).

Received August 1993; accepted December 1993. 